\title{
The TMPRSS6 variant (SNP rs855791) affects iron metabolism and oral iron absorption - a stable iron isotope study in Taiwanese women

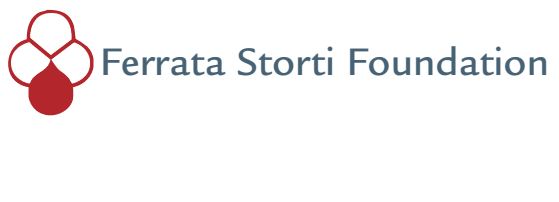

\section{Simone Buerkli, ${ }^{1 *}$ Sung-Nan Pei,,${ }^{2,3,4 *}$ Shu-Chen Hsiao, ${ }^{5}$ Chien-Te Lee, ${ }^{2}$ Christophe Zeder, ${ }^{1}$ Michael B. Zimmermann ${ }^{1}$ and Diego Moretti ${ }^{1}$}

${ }^{1}$ Laboratory of Human Nutrition, Institute of Food Nutrition and Health, Department of Health Science and Technology, Swiss Federal Institute of Technology (ETH Zurich), Zurich, Switzerland; '²Department of Internal Medicine, Kaohsiung Chang Gung Memorial Hospital, Chang Gung University College of Medicine, Kaohsiung, Taiwan; ${ }^{3}$ Department of Hematology Oncology, E-Da Cancer Hospital, Kaohsiung, Taiwan; ${ }^{4}$ College of Medicine, I-Shou University, Kaohsiung, Taiwan and ${ }^{5}$ Department of Pharmacy, Kaohsiung Chang Gung Memorial Hospital, Kaohsiung, Taiwan

${ }^{\circ}$ Current address: Swiss Distance University of Applied Sciences, Department of Health, Regensdorf/Zurich, Switzerland.

*SB and SNP contributed equally as co-first authors.

\section{ABSTRACT}

$\checkmark$ enome wide studies have associated TMPRSS6 rs855791 (2321 C>T) with iron status and hepcidin. It is unclear whether this polymorphism affects iron absorption. We administered standardized ricebased test meals containing $4 \mathrm{mg}$ of labeled ${ }^{57} \mathrm{Fe}$ or ${ }^{58} \mathrm{Fe}$ as $\mathrm{FeSO}_{4}$ on alternate days in non-anemic Taiwanese women ( $\mathrm{n}=79$, 44 TT variant, 35 CC variant). Fractional iron absorption was measured by erythrocyte incorporation of the tracers 14 days after administration. Compared to the CC variant, iron and transferrin saturation were lower $(P=0.001 ; P<0.001$, respectively) and serum hepcidin/transferrin saturation and serum hepcidin/serum iron ratios were higher $(P=0.042 ; P=0.088$, respectively) in the TT variant. Serum hepcidin did not differ between the groups $(P=0.862)$. Geometric mean ( $95 \%$ Confidence Interval [CI]) fractional iron absorption, corrected to a serum ferritin of 15 $\mu \mathrm{g} / \mathrm{L}$, was $26.6 \%$ (95\% CI: $24.0-29.5)$ in the CC variant and $18.5 \%$ (95\% CI: 16.2-21.1) in the TT variant $(P=0.002)$. Overall, predictors of iron absorption were: serum ferritin $(P<0.001)$; genetic variant $(P=0.032)$; and hepcidin $(P<0.001)$. In the models by variant, in the $\mathrm{CC}$ variant the model explained $67-$ $71 \%$ of variability in absorption and serum ferritin was the only significant predictor $(P<0.001)$; while in the TT variant, the model explained only 35$43 \%$ of variability, and hemoglobin $(P=0.032)$, soluble transferrin receptor $(P=0.004)$ and hepcidin $(P<0.001)$ were significant predictors. Women with the TMPRSS6 rs855791 (2321 C>T) polymorphism show altered iron homeostasis which affects oral iron absorption and may increase their risk for iron deficiency. The trial was registered as clinicaltrials gov. Identifier: NCT03317873, and funded by the Kaohsiung Chang-Gung Memorial Hospital, Kaohsiung, Taiwan, (grant CMRPG8F0721) and ETH Zurich, Switzerland.

\section{Introduction}

In the absence of a physiological iron excretion mechanism, long-term iron balance in humans is determined by dietary iron absorption. Systemically, iron absorption is controlled by hepcidin (Hep), a peptide hormone synthesized in hepatocytes ${ }^{1}$ that regulates iron export from cells via its interaction with ferroportin. ${ }^{2}$ Hep is synthesized in response to increasing body iron in a homeostatic feedback loop, involving iron sensing of iron-saturated transferrin by transferrin receptors (Tfr1 and Tfr2) and associated proteins (HFE, hemojuvelin) initiating a cascade involving bone morphogenic protein (BMP) receptor activation. ${ }^{3,4}$

The TMPRSS6 gene encodes the transmembrane serine protease matriptase-2, which interacts with hemojuvelin, modulating the Hep activation pathway. ${ }^{5}$ Consistently with this regulatory model, nonsense mutations in TMPRSS6 cause
Haematologica 2021

Volume 106(11):2897-2905

\section{Correspondence:}

DIEGO MORETTI

diego.moretti@alumni.ethz.ch

Received: June 25, 2020.

Accepted: September 23, 2020.

Pre-published: October 5, 2020.

https://doi.org/10.3324/haematol.2020.264556

(C)2021 Ferrata Storti Foundation

Material published in Haematologica is covered by copyright. All rights are reserved to the Ferrata Storti Foundation. Use of published material is allowed under the following terms and conditions:

https://creativecommons.org/licenses/by-nc/4.0/legalcode. Copies of published material are allowed for personal or internal use. Sharing published material for non-commercial purposes is subject to the following conditions:

https://creativecommons.org/licenses/by-nc/4.0/legalcode, sect. 3. Reproducing and sharing published material for commercial purposes is not allowed without permission in writing from the publisher. 
iron refractory iron deficiency anemia (IRIDA), due to inappropriately elevated Hep levels. ${ }^{6}$ The ratio of serum Hep/transferrin saturation (Hep/TS) may be useful to differentiate subjects with IRIDA from subjects with chronic iron deficiency (ID), ${ }^{7}$ consistent with a disrupted feedback loop between TS and Hep.

Common genetic variants of TMPRSS6, are associated with erythrocyte parameters in human genome wide association studies. ${ }^{8-11}$ The single nucleotide polymorphism (SNP) rs855791 (2321 C>T) of TMPRSS6 has a population frequency of $\approx 0.5$ in Caucasians, ${ }^{10,12} \approx 0.6$ in Japanese $^{13}$ and $\approx 0.2-0.1$ in African Americans. ${ }^{10,12}$ It causes a non-synonymous substitution near the catalytic and active site of the protease, ${ }^{10}$ with a strong association with iron status, erythrocyte parameters, , $^{8-10,12,14-17} \mathrm{Hep}^{18}$ and ratios of Hep to iron indices. ${ }^{19,20}$ T-allele variants in the rs855791 are associated with an increased risk for ID and iron deficiency anemia (IDA). ${ }^{16,17}$ In a case control study in Taiwan, homozygotes for the SNP rs855791 CC had a lower prevalence of IDA, compared to subjects with the CT or TT variant. ${ }^{21}$ In European populations, variants (TT) in rs855791 are associated with lower TS and serum ferritin (SF), higher Hep, and higher ratios of Hep to iron indices. ${ }^{18-20}$ In first time blood donors, the TT variant was associated with larger decreases in SF and hemoglobin $(\mathrm{Hb})$ after multiple donations, suggesting an impaired capacity to replenish stores following donation. ${ }^{22}$

ID is considered the most prevalent nutritional deficiency worldwide and one of the leading causes of anemia among non-pregnant and pregnant women. ${ }^{23}$ While iron status and dietary composition are the main determinants of iron absorption, individual factors other than iron status have been estimated to account for $\approx 50 \%$ of the variance in iron absorption. ${ }^{24}$ Furthermore, a strong familial tendency in iron absorption has been reported in mother child pairs using stable iron isotopes; ${ }^{25,26}$ this could be due to genetic, epigenetic or shared environmental mechanisms.

The genetic determinants of iron status and Hep metabolism in humans, including the effect of mutations in TMPRSS6, are poorly understood. The study aim was to compare iron absorption, Hep and other indices of iron metabolism in iron-sufficient Taiwanese women carrying the TT or the CC variant of the rs855791 SNP in the TMPRSS6 gene. We hypothesized that the TT variant would be associated with higher serum Hep concentrations, higher ratios of Hep to iron indices, and lower iron absorption at comparable iron status.

\section{Methods}

\section{Subjects}

The study was performed at the Kaohsiung Chang Gung Memorial Hospital (K-CGMH) in Taiwan, between February 2018 and February 2019. The flow of study participants is shown Figure 1 . We invited apparently healthy females, with no known history of thalassemia or anemia, aged between 20 to 45 years for screening, assessed their medical history and measured body weight and height, complete blood count, SF and the rs855791 genotype. Inclusion criteria are described in the Online Supplementary Appendix. All participants that were homozygous in the rs855791 (TT or CC), and fulfilled all inclusion criteria were recalled 1 week before the first test meal administration, where we assessed $\mathrm{Hb}$, SF, C-reactive protein (CRP), and menstrual blood losses. Study inclusion criteria were: i) $\mathrm{Hb}>120 \mathrm{~g} / \mathrm{L}$; ii) SF $30-120 \mu g / L$ and iii) CRP $<5 \mathrm{mg} / \mathrm{L}$. The ethical committees of ETH Zurich in Switzerland and the Chang Gung Memorial Foundation Institutional Review Board in Taiwan approved the study. All participants provided written informed consent, and the study was registered as clinicaltrials gov. Identifier: NCT03317873.

On study days 1 and 3 (D1, D3), we administered two standardized rice test meals to fasting participants, labeled with $4 \mathrm{mg}$ iron $\left({ }^{57} \mathrm{Fe}\right.$, and $\left.{ }^{58} \mathrm{Fe}\right)$ as labeled ferrous sulfate $\left(\mathrm{FeSO}_{4}\right)$. A detailed description of the test meal administration and the preparation of stable iron isotopes, can be found in the Online Supplementary Appendix.

\section{Laboratory analyses}

We determined fractional iron absorption (FIA) based on the shift in the enrichment ratio of stable iron isotopes into the erythrocytes on D17. We performed the analyses by inductively coupled plasma mass spectrometry (MC-ICP-MS, Neptune; Thermo Finnigan) as previously described. ${ }^{27}$ We calculated the amounts of ${ }^{57} \mathrm{Fe}$, and ${ }^{58} \mathrm{Fe}$ isotopic labels in blood on D17 on the basis of the shift in iron isotope ratios and on the estimated amount of iron circulating in the body. ${ }^{28}$ We corrected the FIA for SF to the cutoff for ID $(15 \mu \mathrm{g} / \mathrm{L}){ }^{29}$ and to $50 \mu \mathrm{g} / \mathrm{L}$ as a level representing sufficient iron stores with a modification of the Cook et al. formula, ${ }^{30}$ as described in the Online Supplementary Appendix. Procedures such as the assessment of menstrual blood loss, and laboratory measurements such as genotyping, measurement of erythrocyte parameters, CRP, acute phase protein $\alpha$-1-acid glycoprotein (AGP), SF, serum iron ( $\mathrm{SFe}$ ), total iron-binding capacity (TIBC), Hep, and soluble transferrin receptor (sTfR) are also described in the Online Supplementary Appendix.

\section{Sample size calculation}

We based the sample size calculation on a design with two repeated measurements with a compound symmetry covariance structure. Based on previous studies from the Human Nutrition Laboratory, using log-transformed data, we assumed an intra-individual correlation of 0.7 , and a standard deviation of 0.235 . A difference of $30 \%$ in iron absorption was considered relevant. Therefore, we planned to recruit 40 subjects per variant, with $80 \%$ power and $\alpha=0.05$, it allows two dropouts per group. Due to the imbalanced distribution of the minor allele in the Taiwanese population, and difficulties enrolling the planned number of CC subjects, we made a protocol amendment to include $35 \mathrm{CC}$ and 45 TT subjects. This unbalanced distribution results in an estimated power of $75 \%$.

\section{Data and statistical analysis}

We used IBM SPSS statistics (Version 24) for statistical analysis. After testing for normality, we used log-transformed data further analysis if not normally distributed. Normally distributed data is presented as means \pm standard deviation $(\mathrm{SD})$, transformed normal data as geometric mean with the $95 \%$ Confidence Interval $(95 \% \mathrm{CI})$, non-normal data as median and the interquartile range (IQR). Means or medians of red cell parameters, are based on the concentrations measured on D1. Means, medians, or geometric means of CRP, AGP, SF, SFe, TIBC, TS, sTfR, BIS, Hep, Hep/TS, Hep/SF, and FIA are based on concentrations measured on D1 and D3. We tested between-group differences for i) normally distributed variables using independent samples t-test and for ii) not normally distributed variables using Mann-Whitney $\mathrm{U}$ test, as well as iii) differences in CRP, AGP, SF, SFe, TIBC, TS, sTfR, BIS, Hep, Hep/TS, Hep/SF, and FIA by linear mixed models (LMM), with subjects' code as a random intercept, the corresponding variable as a dependent variable and the genotype as a fixed effect. We 


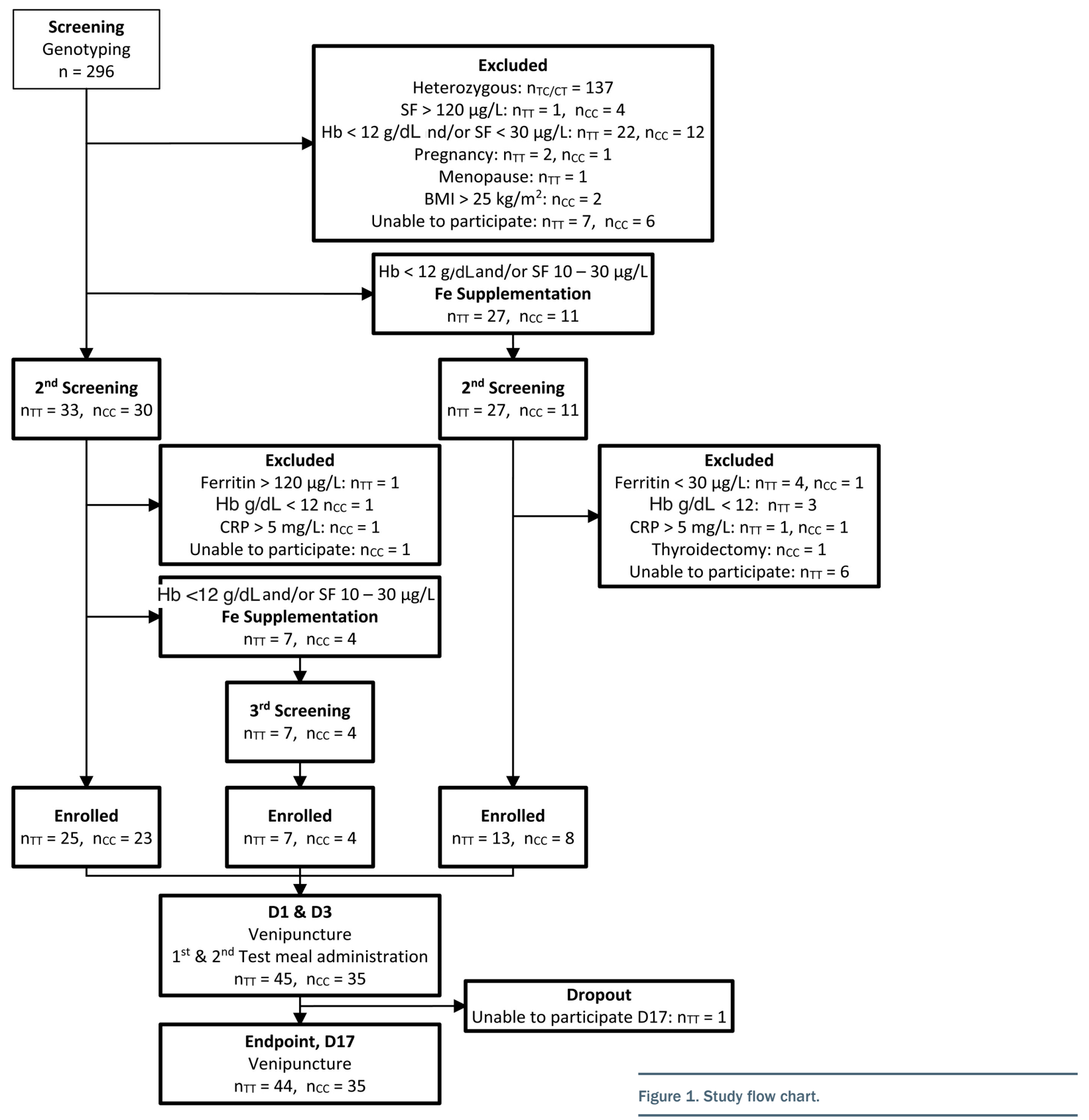

assessed Pearson's correlations and differences between the coefficients with with the Fisher Z-transformation. We assessed predictors of iron absorption with LMM using subjects' code as a random intercept, FIA as a dependent variable, and the genotype, $\mathrm{Hb}$, SF, TS, sTfR, Hep and PBAC as fixed factors. We performed a backward linear regression to assess a minimal adequate model, and we fitted the variables in a LMM. Statistical significance was defined as $P<0.05$.

\section{Results}

\section{Subjects}

We screened 296 women and identified 93 women carrying the TT variant and 66 with the $\mathrm{CC}$ variant, while 137 were excluded as heterozygotes (Figure 1). Of the identified subjects, 33 women with the TT variant and 30 with CC variant met all inclusion criteria. Thirty-four women with TT variants and 15 with CC variants received iron supplements. After the iron supplementation period, 20 subjects with the TT and 12 subjects with the CC variant were included into the study. Finally, 35 subjects with CC and 45 with the TT variant fulfilled all study inclusion criteria and were enrolled (Figure 1). One woman with the TT variant left the study after study D3, thus, 79 women completed the study.

\section{Iron indices}

SF concentrations were balanced between the two variants, while SFe was lower in the TT compared to the CC variant $(P=0.001$; Table 1$)$. Similarly, TS was lower $(P<0.001)$, and TIBC higher $(P=0.086)$ in the TT variant (Table 1). While Hep did not differ between the groups $(P=0.862)$, the Hep/TS ratio $(P=0.042)$ and the Hep/SFe ratio $(P=0.088)$ were $28 \%$ and $25 \%$ higher in the TT variant, respectively (Table 1$)$. None of the subjects had sys- 
Table 1. Subject characteristics of Taiwanese women with the homozygous CC and Tr variants of the rs855791 in TMPRSS6.

\begin{tabular}{|c|c|c|c|}
\hline & CC & $\Pi$ & $P$ \\
\hline$n$ & 35 & 45 & - \\
\hline Age, $y^{*}$ & $34 \pm 6$ & $36 \pm 7$ & $0.436^{\S}$ \\
\hline Weight, kg* & $54.6 \pm 4.8$ & $54.2 \pm 5.2$ & $0.717^{\S}$ \\
\hline Height, cm* & $160 \pm 4$ & $160 \pm 5$ & $0.780^{\S}$ \\
\hline CRP, mg/L $\mathrm{L}^{\dagger}$ & $0.271(0.213,0.346)$ & $0.388(0.306,0.491)$ & $0.125^{\|}$ \\
\hline AGP, g/L* & $0.426 \pm 0.0962$ & $0.455 \pm 0.113$ & $0.206^{\|}$ \\
\hline RBC, million $/ \mu \mathrm{L}^{\ddagger}$ & $4.51(4.19-4.59)$ & $4.58(4.37-4.74)$ & $0.032^{\pi}$ \\
\hline $\mathrm{Hb}, \mathrm{g} / \mathrm{dL}$ & $13.3 \pm 0.6$ & $13.3 \pm 0.7$ & $0.762^{\S}$ \\
\hline HCT, $\%^{\ddagger}$ & $40.0(38.4-40.9)$ & $40.3(38.7-41.5)$ & $0.349^{\mathbb{q}}$ \\
\hline $\mathrm{MCV}, \mathrm{fL} / \mathrm{cell}^{\ddagger}$ & $89.8(88.3-92.0)$ & $88.4(86.4-90.6)$ & $0.008^{\pi}$ \\
\hline MCH, pg/cell ${ }^{\ddagger}$ & $30.0(29.2-31.0)$ & $29.6(28.8-30.0)$ & $0.018^{\pi}$ \\
\hline SF, $\mu \mathrm{g} / \mathrm{L}^{\dagger}$ & $45.1(41.0,49.7)$ & $47.0(43.5,50.9)$ & $0.626^{\|}$ \\
\hline $\mathrm{SFe}, \mu \mathrm{g} / \mathrm{dL}^{\dagger}$ & $114.5(105.2,124.7)$ & $90.3(82.6,98.7)$ & $0.001^{11}$ \\
\hline TIBC, $\mu \mathrm{g} / \mathrm{dL}$ & $315.6 \pm 29.7$ & $327.3 \pm 33.5$ & $\left.0.086\right|^{\|}$ \\
\hline $\mathrm{TS}, \%^{\dagger}$ & $36.5(33.3,39.9)$ & $27.7(25.5,30.2)$ & $<0.001^{11}$ \\
\hline sTfR, mg/L $\mathrm{L}^{\dagger}$ & $4.14(3.97,4.32)$ & $4.33(4.12,4.55)$ & $0.351^{11}$ \\
\hline BIS, mg/kg BW & $7.13 \pm 1.62$ & $7.12 \pm 1.80$ & $0.978^{\prime \prime}$ \\
\hline Hep, $\mathrm{nM}^{\dagger}$ & $2.10(1.80,2.46)$ & $2.06(1.79,2.37)$ & $0.862^{\|}$ \\
\hline $\mathrm{Hep} / \mathrm{SFe}, \mathrm{pmol} / \mu \mathrm{g}^{\dagger}$ & $183.4(160.3,209.8)$ & $227.8(193.3,268.4)$ & $0.088^{\prime \prime}$ \\
\hline Hep/TS, pM/\% $\%^{\dagger}$ & $57.6(50.8,65.4)$ & $74.2(63.0,87.4)$ & $0.042^{\|}$ \\
\hline Hep/SF, pmol/ $\mu \mathrm{g}^{\dagger}$ & $46.5(40.7,53.2)$ & $43.7(38.4,49.8)$ & $0.537^{\prime \prime}$ \\
\hline PBAC* & $126 \pm 63$ & $171 \pm 99$ & $0.015^{\S}$ \\
\hline
\end{tabular}

Anthropometrics, red blood cell (RBC) indices and hemoglobin (Hb) were assessed on day (D) 1,inflammation, iron parameters, plasma hepcidin concentration were assessed on D1 and D3.AGP: acute phase protein $\alpha$-1-acid glycoprotein; BIS: body iron stores; CRP: C-reactive protein; Hb: hemoglobin; HCT: hematocrit; Hep: plasma hepcidin; MCH: mean corpuscular hemoglobin; MCV: mean corpuscular volume; PBAC: pictorial blood-loss assessment chart; SF: serum ferritin; Sfe: serum iron; sTfR: soluble transferrin receptor; TS transferrin saturation; y: years. $\star$ Means \pm standard deviation (SD); ${ }^{\dagger}$ geometric means $\left(95 \%\right.$ Confidence Interval [CI]); ${ }^{\ddagger}$ medians (interquartile range [IQR]); ${ }^{\S}$ differences were assessed by two-sided independent $t$-test: Id differences were assessed by fitting linear mixed models with genotype as fixed effect, participants as the random effects, and the corresponding variable as dependent variable; "differences were assessed by Mann-Whitney U test.

temic inflammation, during the study period (Table 1). The menstrual blood loss scores (PBAC) was higher in the TT variant $(P=0.015$, Table 1$)$.

\section{Fractional iron absorption}

The uncorrected FIA on D1 and D3 within variant did not differ (Table 2; Figure 2) but the Pearson's correlation between days (D1 and D3) FIA was stronger in the CC $(\mathrm{r}=0.86)$ than in the TT variant $(\mathrm{r}=0.67$; for both, $P<0.001$; Figure 2). The mean uncorrected FIA of D1 and D3 of the TI variant, was 7.96\% (95\% CI: 6.87-9.22), and in the CC variant was $6.50 \%$ (95\% CI:5.54-7.62) ( $P=0.160$; Figure 2$)$. FIA corrected to a SF concentration of $15 \mu \mathrm{g} / \mathrm{L}$ was significantly lower in the TT $18.5 \%$ (95\% CI: 16.2-21.1), compared to the CC variant $26.6 \%$ (95\% CI: 24.0-29.5) $(P=0.002$; Table 2; Figure 2). When corrected to a SF of $50 \mu \mathrm{g} / \mathrm{L}$, the TT variant had significantly higher FIA than the CC variant: $7.59 \%$ (95\% CI: 6.66-8.66), compared to $5.70 \%$ (95\% CI: 5.15-6.31) $(P=0.012)$.

\section{Correlation of fractional iron absorption, iron indices and hepcidin}

The Pearson's correlation between FIA and SF was more pronounced in the CC variant $(\mathrm{r}=-0.79, P<0.001)$ than in the TT variant $(r=-0.45, P=0.002)$ and there was a difference in the strength of the correlation between groups $(P<0.001$, Figure 3$)$. Fractional absorption was correlated with TS only in the CC variant (CC: $r=-0.45, P=0.006$; TT $\mathrm{r}=-0.14, P=0.360)$ and the correlation coefficients tended to differ $(P=0.070)$. The correlation of FIA with Hep was more pronounced $(P=0.004)$ in the $\mathrm{CC}$ variant (CC: $\mathrm{r}=-$ 0.81, $P<0.001$, TT: $\mathrm{r}=-0.45, P=0.002)$.

\section{Predictors of fractional iron absorption}

Genetic variant $(\beta=-0.346, P=0.032)$ was a significant predictor of overall FIA along with $\operatorname{SF}(\beta=-0.393, P<0.001)$, and Hep $(\beta=-0.312, P<0.001),\left(R_{\text {adjusted }}^{2}=0.468\right)$, Table 3. Stepwise deletion removed TS, PBAC and CRP from the model $\left(R_{\text {adiusted }}^{2}=0.469\right)$. In the prediction model by variant, in the CC variant only SF was significantly associated with FIA ( $\beta=-0.696, P<0.001)$, explaining $67 \%$ of the variability in iron absorption $\left(R_{\text {adiusted }}^{2}=0.669\right)$ (Table 4$)$. In contrast, in the TT variant, $\operatorname{Hep}(\beta=-0.353, P<0.001)$, sTfR $(\beta=0.317$ $P=0.004), \mathrm{Hb}(\beta=-0.252, P=0.023)$, and TS $(\beta=0.199$, $P=0.011$ ) were associated with FIA (Table 4), but with a substantially lower coefficient of determination $\left(R_{\text {adjusted }}^{2}=0.375\right)$, explaining $38 \%$ of the variability. In the minimal adequate model (Table 5), for the CC variant, significant predictors are SF $(\beta=-0.667, P<0.001)$ and Hep $(\beta=-0.217, P=0.002)\left(R_{\text {adiusted }}^{2}=0.688\right)$. For the TT variant, significant predictors are Hep, $(\beta=-0.411, P<0.001)$ sTfR, $(\beta=0.320, \quad P=0.003)$ and $\mathrm{Hb} \quad(\beta=-0.226, \quad P=0.038)$ $\left(R_{\text {adjusted }}^{2}=0.356\right.$, Table 5).

\section{Discussion}

Our study shows that the TMPRSS6 rs855791 TT variant is associated with lower iron absorption in an overall model controlling for other iron status indicators. At a 
Table 2. Fractional iron absorption from rice meals in $\mathrm{CC}$ and $\mathrm{T}$ variants of the rs855791.

$\begin{array}{lccc} & \text { CC variant } & \text { TI variant } & P \\ \mathrm{FIA}_{\mathrm{D} 1}, \% & 6.50(5.14,8.22)^{*} & 7.99(6.39,9.98)^{\dagger} & 0.206^{\ddagger} \\ \mathrm{FIA}_{\mathrm{D} 3}, \% & 6.49(5.17,8.15)^{*} & 7.93(6.50,9.68)^{\dagger} & 0.183^{\ddagger} \\ \mathrm{FIA}_{\mathrm{D} 1} \&_{\mathrm{D} 3}, \% & 6.50(5.54,7.62) & 7.96(6.87,9.22) & 0.160^{\S} \\ \mathrm{FIA}_{\mathrm{D} 1} \&_{\mathrm{D} 3}, \mathrm{SF} 15 \mathrm{corr}, \% \text { ll } & 26.6(24.0,29.5) & 18.5(16.2,21.1) & 0.002^{\S}\end{array}$

Values are the geometric means and the $95 \%$ Confidence Interval (CI). D1: study day 1; D3: study day 3; FIA: fractional iron absorption; SF15corr: serum ferritin correction to a concentration of $15 \mu \mathrm{g} / \mathrm{L}$. *differences between study day one and three were assessed by paired $\mathrm{t}$ test $P=0.984$. $^{\dagger}$ differences between study day one and three were assessed by paired $t$ test $P=0.935$. ${ }^{*}$ differences between the two variants were assessed by two-sided independent $t$-test. ${ }^{\S}$ differences between the two variants were assessed by fitting linear mixed models with genotype as fixed effect, participants as the random effects, and the corresponding variable as dependent variable. Ilcorrection was done using the formula: $\log (\mathrm{FIAC})=\log (\mathrm{FIAO})+\mathrm{a}{ }^{*} \log (\mathrm{SFC} / \mathrm{SFO})$, with $\mathrm{aCC}=-1.28, \mathrm{aTT}=-0.74$.

A

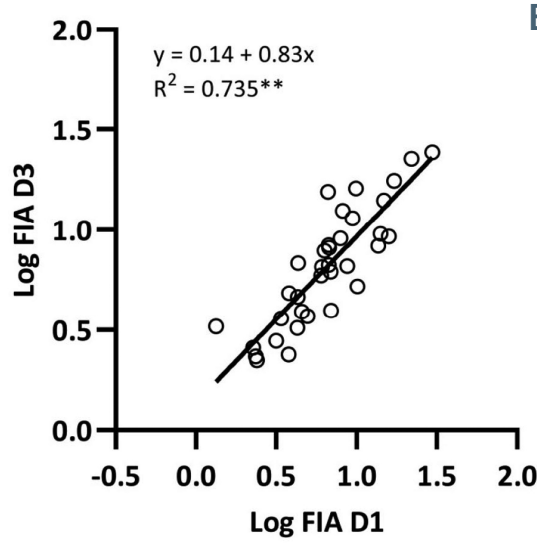

C

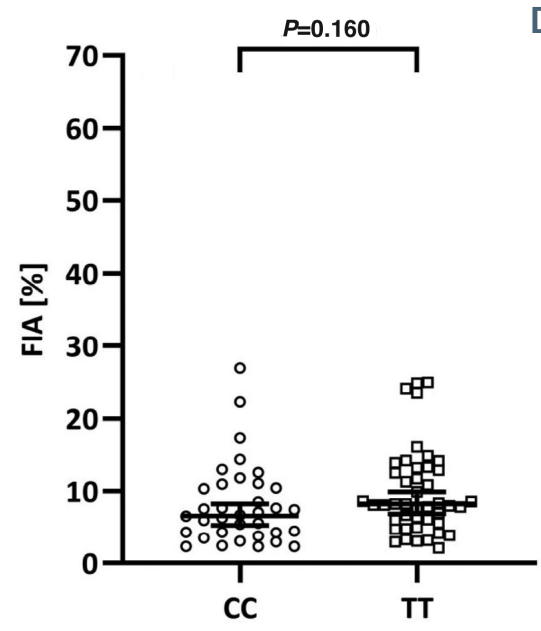

B

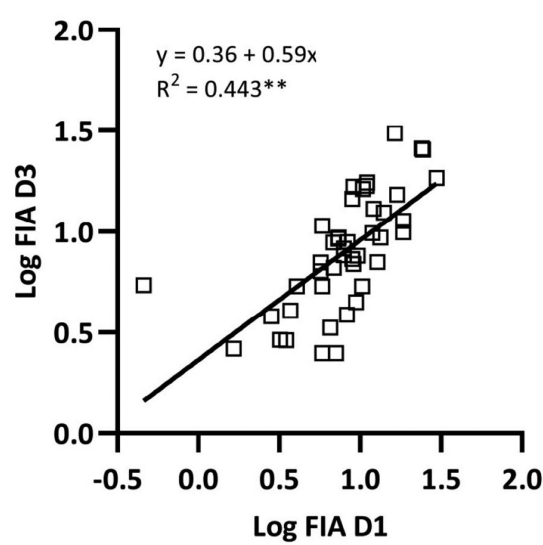

D

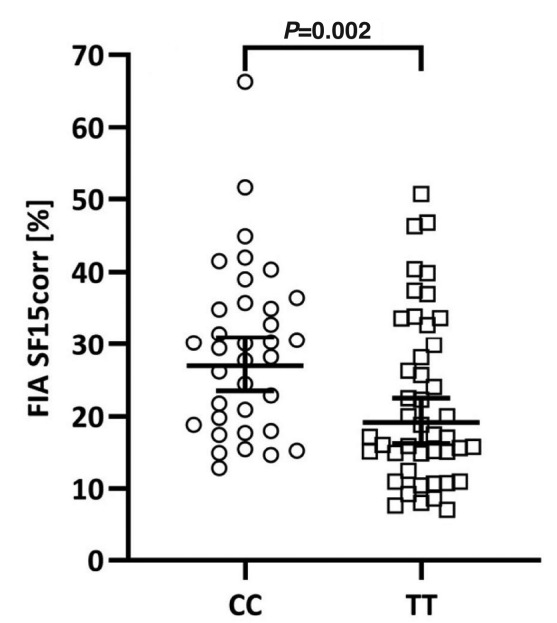

Figure 2. Correlation of the inter-individua fractional iron absorption ( $A$ and $B$ ) and fractional iron absorption (C-D) in rs855791 variants. (A and B) Fractional iron absorption (FIA) measured from identical rice test meals on study day one and three, separated by variant in the TMPRSS6 rs855791, in (A) the $C C$ variant $(\circ n=35)$, and in (B) the $T T$ ( $\mathrm{n}=44)$. The Pearsons correlation factors are: 0.86 , and 0.67 for the $\mathrm{CC}$ and $\mathrm{TT}$, respectively (both, $* * P<0.001)$. ( C and D) Each point represents the mean of the FIA on day 1 (D1) and D3 from two identical rice meals, the line represents the geometric mean and the bars the $95 \%$ Confidence interval $(\mathrm{Cl})$. (C) the measured FIA versus (D) the FIA corrected to a serum ferritin (SF) concentration of 15 $\mu \mathrm{g} / \mathrm{L}$ are shown. Differences betweenthe two variants were assessed by fitting linear mixed models with genotype as fixed effect, participants as the random effects, and FIA or FIA corrected for SF as the dependent variable $\mathrm{CC}(\circ, \mathrm{n}=35)$ versus TT $(\square, \mathrm{n}=44)$. standardized SF concentration of $15 \mu \mathrm{g} / \mathrm{L}$, iron absorption was significantly lower in the TT variant. The TT variant also had lower TS and SFe and higher Hep/TS ratios, suggesting an altered interplay of SFe, hepcidin, iron stores and the regulation of dietary absorption compared to the CC variant. Similarly, known predictors of iron absorption explained much less of the variability in iron absorption in the TT variant. To our knowledge, this is the first study comparing dietary iron absorption using stable iron isotopes among the common SNP rs855791 of the TMPRSS6, which has been associated with iron status and red blood cell parameters in various genome wide association studies and large cross-sectional studies. ${ }^{10,12,15-17}$

In humans, inter-subject absorption of nonheme iron shows a wide variation in healthy young women. Zimmermann et al. reported a variation from 1\% to $58 \%$ in iron absorption from standardized test meals labeled with $4 \mathrm{mg}$ Fe as stable isotopes. ${ }^{31}$ Some of this variation is due to differences in iron status and meal matrix, however, taken together, it has been estimated that iron status and food factors predict only $\approx 50 \%$ of the variance in iron absorption in a population. ${ }^{24}$ Cook et al. ${ }^{32}$ reported a striking positive correlation in body iron in iron-replete mothers and their young children and suggested this close correlation was due to a shared diet and/or possible genetic determinants of iron status such as shared iron-regulatory genes. In Mexican $(n=18)$, and Senegalese mother-child pairs $(n=17)$, non-heme-iron absorption measured with stable isotopes exhibited strong and intermediate correlations, respectively. ${ }^{25,26}$ A common polymorphism in the transferrin protein (G277S) has been associated with ID in American women, ${ }^{33}$ but a stable isotope study comparing 25 iron deficient, non-anemic women who had either a heterozygous G277S/G277G or wild-type G277G/G277G genotype did not find a significant difference in iron absorption. ${ }^{34}$ However, the G277S carriers did not show 
Table 3. Predictors of iron absorption in healthy Taiwanese women $(n=79)$.

\begin{tabular}{|c|c|c|c|c|c|c|}
\hline & \multicolumn{3}{|c|}{ Overall Model* } & \multicolumn{3}{|c|}{ Minimal Adequate Model ${ }^{\dagger}$} \\
\hline Variables & $\beta$ & SE & $P$ & $\beta$ & SE & $P$ \\
\hline Intercept & 0.14 & 0.10 & 0.159 & 0.13 & 0.01 & 0.197 \\
\hline Variant $(\mathrm{CC} \text { us. TT })^{\ddagger}$ & -0.35 & 0.16 & 0.032 & -0.30 & 0.15 & 0.045 \\
\hline Hemoglobin & -0.13 & 0.07 & 0.085 & -0.11 & 0.07 & 0.154 \\
\hline Serum Ferritin & -0.39 & 0.08 & $<0.001$ & -0.41 & 0.07 & $<0.001$ \\
\hline Transferrin Saturation & 0.11 & 0.06 & 0.063 & \multicolumn{3}{|c|}{ removed from the model ${ }^{\S}$} \\
\hline Soluble Transferrin Receptor & 0.12 & 0.07 & 0.083 & 0.12 & 0.07 & 0.105 \\
\hline Plasma Hepcidin & -0.31 & 0.06 & $<0.001$ & -0.29 & 0.06 & $<0.001$ \\
\hline PBAC & 0.07 & 0.08 & 0.389 & \multicolumn{3}{|c|}{ removed from the model ${ }^{\S}$} \\
\hline C-reactive Protein & -0.05 & 0.07 & 0.434 & \multicolumn{3}{|c|}{ removed from the model ${ }^{\S}$} \\
\hline
\end{tabular}

Analyzed by LMM using standardized variables, dependent variable: fractional iron absorption; fixed factors: potential continuous or categorical predictors; random effects: Subjects' code. Shown are standardized $\beta$-coefficients standard errors (SE). Hemoglobin, menstrual blood loss scores (PBAC) were assessed on day (D) 1 , inflammation, iron parameters, plasma hepcidin concentration, and fractional iron absorption (FIA) are based on data measured on D1 and D3. $\star$ Regression model fit: $R^{2}=0.498$; $R_{\text {adjusted }}^{2}=0.468 ;{ }^{\dagger}$ assessed by backward linear regression; regression model fit: $R^{2}=0.486 ; R_{\text {adjusted }}^{2}=0.469 ;$; nominal variable; $1=\mathrm{CC}, 2=\mathrm{TT}$; ${ }^{\S}$ removed variable by the backward regression to assess the minimal adequate model.

Table 4. Potential predictors of iron absorption in variants of the TMPRSS6 rs855791 (nCC=35, nT=44).

\begin{tabular}{lcccccc} 
& & CC Variant* & & \multicolumn{3}{c}{ TIVariant } \\
Variables & $\beta$ & SE & $P$ & $\beta$ & SE & $P$ \\
Intercept & 0.0003 & 0.08 & 0.997 & -0.02 & 0.10 & 0.851 \\
\hline Hemoglobin & 0.05 & 0.09 & 0.559 & -0.25 & 0.11 & 0.023 \\
Serum Ferritin & -0.70 & 0.010 & $<0.001$ & -0.20 & 0.11 & 0.060 \\
\hline Transferrin Saturation & -0.02 & 0.07 & 0.752 & 0.20 & 0.08 & 0.011 \\
Soluble Transferrin Receptor & -0.12 & 0.08 & 0.161 & 0.32 & 0.10 & 0.004 \\
\hline Plasma Hepcidin & -0.16 & 0.08 & 0.053 & -0.35 & 0.08 & $<0.001$ \\
PBAC & -0.12 & 0.09 & 0.229 & 0.14 & 0.11 & 0.211 \\
\hline C-reactive Protein & 0.04 & 0.08 & 0.614 & -0.05 & 0.10 & 0.610
\end{tabular}

Analyzed by LMM using standardized variables, dependent variable: fractional iron absorption; fixed factors: potential continuous or categorical predictors; random effects: Subjects' code. Shown are standardized $\beta$-coefficients with their standard errors. Hemoglobin, menstrual blood loss scores (PBAC) were assessed on day (D) 1 , inflammation, iron parameters, plasma hepcidin concentration, and fractional iron absorption are based on data measured on D1 and D3. *Regression model fit of CC variant: $R^{2}=0.707$; $R_{\text {adjusted }}^{2}=0.669 ;{ }^{\dagger}$ regression model fit of TT variant: $R^{2}=0.432 ; R_{\text {adjusted }}^{2}=0.375$.

the typical inverse correlation between iron absorption and $\mathrm{SF}^{34}$ Similarly, in our study with the TMPRSS6 rs855791 mutation, in the TT variant, the correlation between iron absorption and SF, and iron absorption and Hep, were only weak and moderate, respectively. In contrast to the CC variant, where both these correlations are strong. Also the models computed by variant show remarkable differences. In the CC variant SF alone is significantly associated with FIA, explaining $67 \%$ of the variability. In the TT variant, in contrast, several factors identify as being associated with FIA: Hep, sTfR, $\mathrm{Hb}$, and TS, and their total contribution explain only $38 \%$ of the variability in iron absorption.

Our hypothesis that the CC variant would have increased iron absorption was based on a regulatory model of TMPRSS6 acting as a negative regulator of the Hep activation pathway, and we hypothesized the largest effects would be seen in an iron replete population, where Hep expression would be activated. However, our findings indicate the effects of the genetic variant are likely most relevant at low iron status (Figure 3); at lower SF, women with the TT variant were less able to upregulate iron absorption, which could increase the risk for ID. Further, the overall model (Table 3) shows that iron status indices, Hep, and genotype, but not inflammation and menstrual blood loss are associated with fractional iron absorption. A recent large study in blood donors suggests an impaired capacity in the TT variant to replenish iron stores after repeated blood donations, even if the possibly protective CC variant was not enriched in high intensity donors. ${ }^{22}$ It is also possible that cellular mechanisms controlled by iron regulatory proteins are, especially at intermediate serum iron levels, able to compensate for the altered interplay of Hep and TS in the TT variant by inducing the translation of iron transporters (e.g., DMT1) and transcription factor HIF-2 $\alpha .^{35}$ Such a compensatory mechanism was suggested in a recent study in women in whom an acute inflammatory stimulus increased Hep but did not affect iron absorption. ${ }^{36}$

Our findings suggest that, at low SF concentrations, women with the TI variant have lower iron absorption, whereas when iron stores are replete, they may be less able to downregulate iron absorption compared to the CC variant. Our variant-specific FIA correction to SF uses a similar approach as the original formula of Cook et al..$^{30}$ used to correct dietary absorption measurements for the individual iron status; that formula employs a slope of -1 between log FIA and log SF. We propose adapted, regression formulas with slopes of -1.28 and -0.74 for CC and TT variants, respectively (Figure 3 ).

In a case control study in Taiwanese women comparing women with IDA to non-anemic controls, the CC variant was less frequent in the IDA group compared to the control group (12\% vs. $25 \%)$; this suggests the CC variant 
A

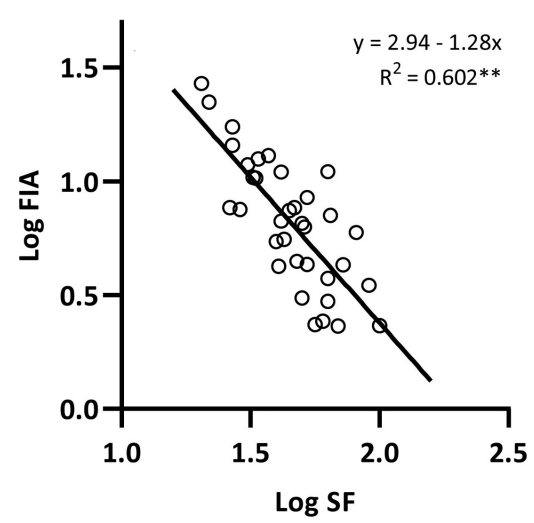

C

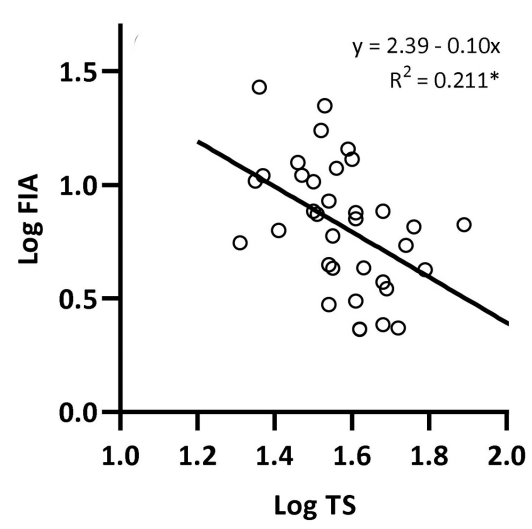

E

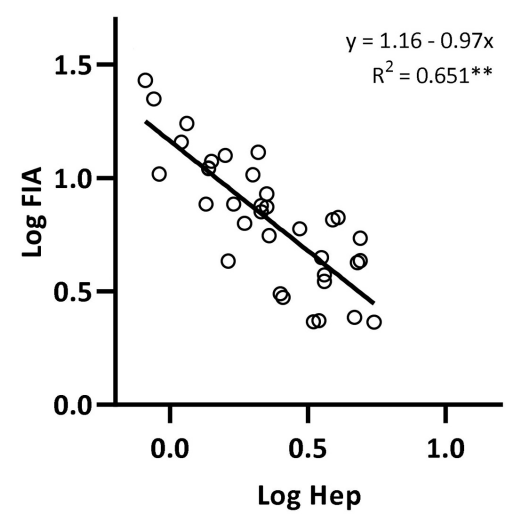

B

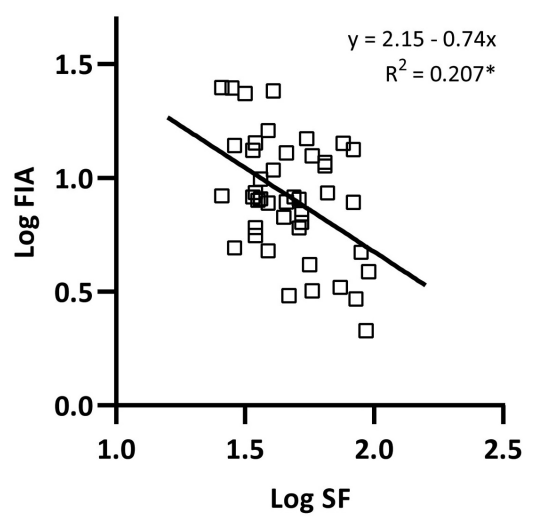

D
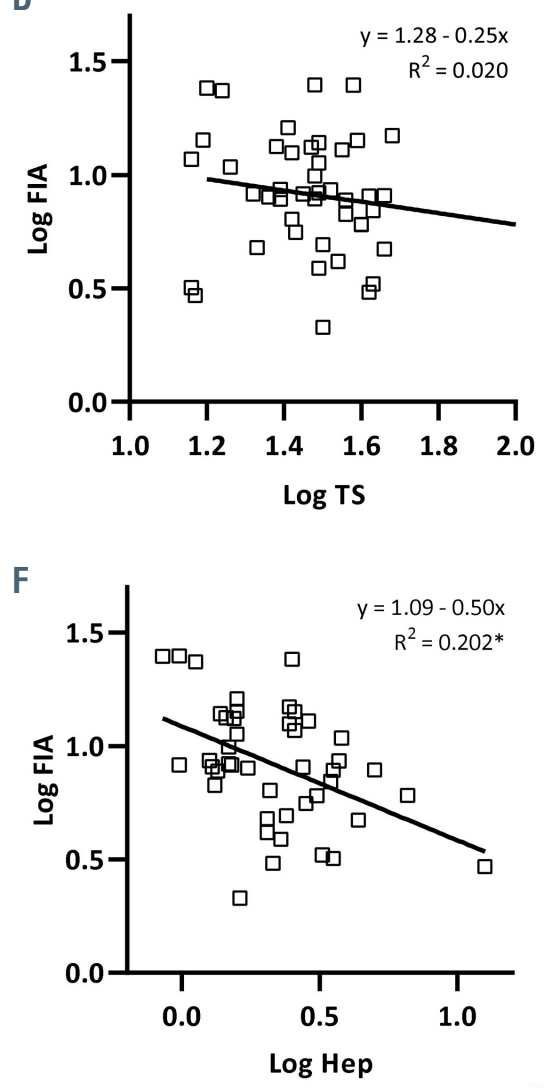

Figure 3. Correlations of fractional iron absorption. Correlations between fractional iron absorption (FIA) and serum ferritin (SF) (A and B), transferrin saturation (TS) (C and D) and hepcidin (Hep) (E and F) of the participants separated.by the variants in the TMPRSS6 rs855791, CC ( $\circ, n=35)$ and TT ( $\square, n=44)$. Each point represents one participant and their mean of FIA, SF, TS, and Hep measured on study day 1 (D1) and D3. Pearson's correlation factors $r$ for FIA to SF correlation are: $-0.79 \quad(P<0.001)$ and -0.45 $(P=0.002)$; for FIA to TS correlation: -0.45 $(P=0.006)$ and $-0.14(P=0.360)$; for FIA to Hep correlation: $-0.81(P<0.001)$ and 0.45 , $(P=0.002)$, for the $C C$ variants and TT variants respectively. $* * P<0.001$, $\star P<0.05$, may reduce risk of IDA. ${ }^{21}$ This effect is also suggested in our screening data: among the screened subjects, $60 \%$ of women with the TT variant had either Hb below $12 \mathrm{~g} / \mathrm{dL}$ and/or SF below the study inclusion criteria, compared to $42 \%$ of women with the CC variant. Also, among women who received iron supplementation due to ID, $80 \%$ of the women with the CC variant replenished their iron stores, in contrast to only $59 \%$ of women with the TT variant. While this is consistent with the view that women with the TT variant are at a higher risk for ID and may have a blunted response to iron supplements when body iron stores are low, this hypothesis needs confirmation in larger prospective trials. Further mechanistic studies in monozygotic twins would be particularly informative as they may distinguish potential genetic and epigenetic sources of variability in iron absorption.

Our findings are consistent with previous studies that have shown that Sfe and transferrin saturation are lower, and TIBC is higher in the TT variant. ${ }^{18-20}$ The higher Hep/TS ratio reported in women with the TT variant in our study has been previously described in Italian ${ }^{18}$ and Dutch populations. ${ }^{20}$ In our study, the lack of association between variant and Hep suggests a different modulation of iron regulatory signals (transferrin bound iron and iron stores) in the regulation of Hep between the two different variants. Consistent with this interpretation, a recent study has suggested that the Hep/TS ratio may be a useful diagnostic marker to differentiate IRIDA patients from those with chronic ID. ${ }^{7}$

A strength of this study is that iron absorption was assessed from an isotopically labeled standardized labeled test meals in a relatively large number of subjects, using erythrocyte incorporation of stable iron isotope labels. Due to its precision, this approach allows, in combination with iron indices, to study regulatory aspects of iron metabolism in humans. ${ }^{36,37}$ We performed iron absorption 
Table 5. The minimal adequate model and predictors of iron absorption in variants of the TMPRSS6 rs $855791\left(\mathrm{n}_{\mathrm{cC}}=35, \mathrm{n}_{\pi}=44\right)$.

\begin{tabular}{|c|c|c|c|c|c|c|}
\hline & & CC variant* & & & II Variant' & \\
\hline Variables & $\beta$ & SE & $P$ & $\beta$ & SE & $P$ \\
\hline Intercept & 0.0004 & 0.08 & 0.996 & -0.01 & 0.11 & 0.911 \\
\hline Hemoglobin & & removed from the model ${ }^{+}$ & -0.23 & 0.11 & 0.038 & \\
\hline Serum Ferritin & -0.67 & 0.09 & $<0.001$ & & removed from the model ${ }^{\ddagger}$ & \\
\hline Transferrin Saturation & & removed from the model ${ }^{+}$ & & & removed from the model ${ }^{\ddagger}$ & \\
\hline Soluble Transferrin Receptor & & removed from the model ${ }^{\ddagger}$ & 0.32 & 0.10 & 0.003 & \\
\hline Plasma Hepcidin & -0.22 & 0.07 & 0.002 & -0.41 & 0.08 & $<0.001$ \\
\hline PBAC & -0.11 & 0.09 & 0.226 & & removed from the model ${ }^{\ddagger}$ & \\
\hline C-reactive protein & & removed from the model & & & removed from the model ${ }^{*}$ & \\
\hline
\end{tabular}

The minimal adequate model is assessed by backward linear regression using standardized variables. Parameters shown are analyzed by LMM, dependent variable: fractional iron absorption; fixed factors: potential continuous or categorical predictors; random effects: Subjects' code. Shown are standardized $\beta$-coefficients with their standard errors (SE). Hemoglobin, menstrual blood loss scores (PBAC) were assessed on day (D) 1, inflammation, iron parameters, plasma hepcidin concentration, and fractional iron absorption are based on data measured on D1 and D3. *Regression model fit of CC variant: $R^{2}=0.702 ; R^{2}{ }_{\text {adjustec }}=0.688$; $\dagger$ regression model fit of TT variant: $R^{2}=0.378 ; R^{2}$ $\ddagger$ removed variable by the backward regression to assess the minimal adequate model.

measurements twice in each subject; this increased statistical power and allowed us to make intra-individual comparisons. Our study also has limitations: our assessment of menstrual loss using PBAC is semi-quantitative, and while we found no association with iron absorption after correcting for iron status in the overall model, we cannot fully exclude a potential effect of menstrual blood loss on iron absorption. Our proposed genotype-specific slopes of SF and iron absorption are based on a relatively narrow range of iron status and should be studied in populations with broader iron status distribution. We focused our hypothesis on a single SNP, and we did not study the interplay with other SNP known to affect iron homeostasis. However, the HFE rs1800562 (C282Y) mutation is known to be rare in Taiwanese women. ${ }^{38}$ In contrast, the GNPAT rs11558492 has been associated with a high-iron phenotype, ${ }^{39}$ and a recent study in Taiwanese women has shown a minor allele frequency of $12 \%$, and a significant higher serum iron response after a supplement. ${ }^{40}$ We did not study heterozygotes, despite the fact that effects on iron absorption are conceivable in this group. Furthermore, unknown SNP associated with rs855791 may explain the observed effects. However, we think this possibility is unlikely, as rs855791 has been repeatedly shown to be associated with iron status, as discussed above.

To summarize, we have shown that in a fully adjusted model of iron absorption, women with the TT variant have lower iron absorption compared to women with the $\mathrm{CC}$ variant. This may be associated with higher Hep/TS and Hep/SFe ratios, suggesting impaired negative feedback on Hep synthesis by circulating iron. Furthermore, in the TT variant, regulation of iron absorption is less well predicted by iron stores. Thus, our findings suggest women with the TT variant are less able to upregulate iron absorption at low iron status, which may increase their risk of ID.

\section{Disclosures \\ No conflicts of interest to disclose.}

\section{Acknowledgments}

The authors thank all subjects who participated in the study and the nursing staff who essentially contributed to the conduction; MiaoChin Sun and Yu-Ching Chan for preparation of all the test meals, help in participant recruitment and study conduction; Min-Yi Tsai for sample handling and genetic variant analysis; Nicole Härter for careful preparation of the whole blood samples; Adam Krzystek, and Timo Christ for careful analysis of the samples on the MS-ICPMS.

\section{Contributions}

$M B Z, D M, S B$, and SNP designed the study; SNP, SCH and $C T L$ conducted the study and collected the samples; $S B$ and $C Z$ analyzed the samples and performed the statistical analyses, $S B$, $D M, M B Z$, and SNP participated in the data interpretation; $S B$ wrote the first draft of the manuscript; all authors edited the manuscript and approved the final version.

\section{Funding}

This study was supported by the Kaohsiung Chang-Gung Memorial Hospital, Kaohsiung, Taiwan, (grant CMRPG8F0721) and Laboratory of Human Nutrition, Institute of Food Nutrition and Health, Department of Health Science and Technology, Swiss Federal Institute of Technology (ETH Zurich), Zurich, Switzerland.

\section{References}

1. Zumerle S, Mathieu JR, Delga S, et al. Targeted disruption of hepcidin in the liver recapitulates the hemochromatotic phenotype. Blood. 2014;123(23):3646-3650.

2. Nemeth E, Tuttle MS, Powelson J, et al. Hepcidin regulates cellular iron efflux by binding to ferroportin and inducing its internalization. Science. 2004;306(5704): 2090-2093.

3. Gao J, Chen J, Kramer M, Tsukamoto H, Zhang AS, Enns CA. Interaction of the hereditary hemochromatosis protein HFE with transferrin receptor 2 is required for transferrin-induced hepcidin expression. Cell Metab. 2009;9(3):217-227.

4. Steinbicker AU, Bartnikas TB, Lohmeyer LK, et al. Perturbation of hepcidin expression by BMP type I receptor deletion induces iron overload in mice. Blood. 2011; 118(15):4224-4230.

5. Silvestri L, Pagani A, Nai A, De Domenico I, Kaplan J, Camaschella C. The serine protease matriptase-2 (TMPRSS6) inhibits hepcidin activation by cleaving membrane hemojuvelin. Cell Metab. 2008;8(6):502511.
6. Camaschella C. New insights into iron deficiency and iron deficiency anemia. Blood Rev. 2017;31(4):225-233

7. Heeney MM, Guo D, De Falco L, et al. Normalizing hepcidin predicts TMPRSS6 mutation status in patients with chronic iron deficiency. Blood. 2018;132(4):448 452.

8. McLachlan S, Giambartolomei C, White J, et al. Replication and characterization of association between $\mathrm{ABO}$ SNPs and red blood cell traits by meta-analysis in Europeans. PLoS One. 2016;11(6): e0156914. 


$$
\text { Th }
$$

